Revista de Matemática: Teoría y Aplicaciones 2007 14(1) : 65-80

CIMPA - UCR - CCSS ISSN: 1409-2433

\title{
CARACTERIZACIÓN DE ESPACIOS DE BANACH MEDIANTE DESIGUALDADES Y EL MAPEO DE DUALIDAD*
}

\author{
WiLLIAM J. UGALDE ${ }^{\dagger}$
}

Recibido/Received: 16 Nov 2006 - Aceptado/Accepted: 31 Jan 2007

\begin{abstract}
Resumen
Usando un resultado de C. E. Chidume, presentamos una prueba mejorada y más corta de un resultado de $\mathrm{Xu}$ y Roach sobre caracterización de espacios de Banach uniformemente convexos y uniformemente suaves mediante el mapeo de dualidad y desigualdades que involucran funciones estríctamente crecientes y estríctamente decrecientes.
\end{abstract}

Palabras clave: espacios de Banach, mapeos de dualidad, espacios uniformemente convexos, espacios uniformemente suaves, módulo de convexidad, módulo de suavidad.

\begin{abstract}
Using a result of C. E. Chidume, we present a shorter and improved proof of a result of Xu and Roach on characterization of uniformly convex and uniformly smooth Banach spaces by the duality map and inequalities involving strictly increasing and strictly decreasing functions.
\end{abstract}

Keywords: Banach spaces, duality maps, uniformly convex spaces, uniformly smooth spaces, modulus of convexity, modulus of smoothness.

Mathematics Subject Classification: 46B25, 46B20.

\footnotetext{
${ }^{*}$ Research supported in part by The Abdus Salam International Center for Theoretical Physics and by Oficina de Asuntos Internacionales de la Universidad de Costa Rica.

${ }^{\dagger}$ Department of Mathematics, University of Costa Rica, 2060 San José, Costa Rica. E-Mail: wugalde@cariari.ucr.ac.cr
} 


\section{Introducción}

Uno de los principales papeles del producto interno en un espacio de Hilbert $H$ es el permitir interpretar un elemento $x$ en $H$ como un funcional $x^{*}$ sobre $H$ con las propiedades $\left\|x^{*}\right\|^{2}=\|x\|^{2}=\left\langle x^{*} \mid x\right\rangle$. En general, la importancia del producto interior en el estudio de fenómenos que toman lugar entre espacios de Hilbert es invaluable. Por otro lado, muchos modelos matemáticos no viven en forma natural en espacios de Hilbert. Con el fin de buscar sustitutos de las herramientas de espacios de Hilbert en el estudio de la geometría de espacios de Banach, en 1962 Beurling y Livingston [1] intrudujeron el mapeo normalizado de dualidad

$$
J: x \in X \rightarrow\left\{x^{*} \in X^{*}:\left\|x^{*}\right\|^{2}=\|x\|^{2}=\left\langle x^{*} \mid x\right\rangle\right\}
$$

el cual reemplaza el isomorphismo $H \sim H^{*}$. Note que $J$ no es vacío gracias al Teorema de Hahn-Banach.

En un espacio $H$ con producto interno, una importante herramienta geométrica es la ley del paralelogramo $\|x+y\|^{2}+\|x-y\|^{2}=2\left(\|x\|^{2}+\|y\|^{2}\right)$. En el caso particular $\|x\|=\|y\|=1$ se tiene $\|(x+y) / 2\|^{2}=1-\|x-y\|^{2} / 4$. De esta desigualdad se puede determinar la distancia entre el punto medio del segmento que une $x$ y y y la esfera $S=\{x \in H:\|x\|=1\}$ en $H$ mediante $1-\|(x+y) / 2\|=1-\sqrt{1-\|x-y\|^{2} / 4}$. Evidentemente esta distancia está siempre entre 0 y 1 , además si $\varepsilon \leq\|x-y\|$ entonces

$1-\|x+y\| / 2 \leq 1-\sqrt{1-\varepsilon^{2} / 4}$. La idea detrás de esta fórmula es la convexidad de la bola unidad en un espacio con producto interior: si la distancia entre dos puntos $x$ y $y$ en la esfera unidad es mayor que $\varepsilon$ entonces el punto medio del segmento que une $x$ y $y$ permanece dentro de la bola unidad con $1-\varepsilon^{2} / 4 \leq\|(x+y) / 2\|^{2}$. De este modo, es posible estudiar qué tan convexa es la bola unidad en espacios que en lugar de poseer un producto interno poseen simplemente una norma.

Un espacio de Banach se dice ser redondo o estríctamente convexo si las igualdades $\|x\|=\|y\|=\|x+y\| / 2=1$ implican $x=y$. Con el fin de entender la "redondez" de un espacio normado $X$, con $\operatorname{dim} X \geq 2$, estudiamos su módulo de convexidad $\delta_{X}:(0,2] \rightarrow$ $[0,1]$

$$
\delta_{X}(\varepsilon)=\inf \left\{1-\frac{\|x+y\|}{2}:\|x\| \leq\|y\| \leq 1, \varepsilon \leq\|x-y\|\right\} .
$$

En el caso particular de un espacio de Hilbert $H$ se tiene $\delta_{H}(\varepsilon)=1-\sqrt{1-\varepsilon^{2} / 4}$.

Un espacio de Banach es uniformemente convexo si su módulo de convexidad es positivo, es decir, "si dos puntos en la bola unitaria de un espacio uniformemente convexo están aparte, entonces su punto medio se encuentra dentro de la bola." Particularmente útil para el presente trabajo es el siguiente resultado cuya prueba se puede leer en [3].

Teorema 1. El módulo de convexidad de un espacio de Banach $X$ es una función convexa y continua.

Los mapeos de dualidad buscan construir el puente para pasar entre los espacios de Banach uniformemente convexos y los espacios de Banach uniformemente suaves. En particular permiten concluir que un espacio $X$ es uniformente suave si y solo si $X^{*}$ es 
uniformemente convexo y $X$ es uniformemente convexo si y solo si $X^{*}$ es uniformemente suave.

Nuestro objetivo es presentar una prueba más corta y mejorada de la caracterización de espacios uniformemente convexos y uniformemente suaves de Xu y Roach [9] usando el siguiente resultado de Chidume [3] sobre espacios uniformemente convexos.

Teorema 2. Un espacio $X$ es uniformemente convexo si y solo si para cada $1<p<\infty$, existe una función estríctamente creciente $s(p, \lambda, \cdot): \mathbb{R}^{+} \rightarrow \mathbb{R}^{+}$tal que $s(p, \lambda, 0)=0$ y para toda $x, y$ en $X$ y $\lambda$ en $[0,1]$ se tiene

$$
\|\lambda x+(1-\lambda) y\|^{p}+(\max \{\|x\|,\|y\|\})^{p} s\left(p, \lambda, \frac{\|x-y\|}{\max \{\|x\|,\|y\|\}}\right) \leq \lambda\|x\|^{p}+(1-\lambda)\|y\|^{p} .
$$

Además,

$$
K_{p} \delta_{X}(\varepsilon / 2) \leq \lim \sup _{\lambda \rightarrow 0} \frac{s(p, \lambda, \varepsilon)}{\lambda}+\limsup _{\lambda \rightarrow 1} \frac{s(p, \lambda, \varepsilon)}{1-\lambda}
$$

donde $K_{p}$ es la constante positiva definida por

$$
\begin{aligned}
K_{p}= & 4(2+\sqrt{3}) \min \left\{\min \left\{\frac{p(p-1)}{2}, 1\right\},(p-1) \min \left\{\frac{p}{2}, 1\right\},\right. \\
& \left.(p-1)\left(1-(\sqrt{3}-1)^{\frac{p}{p-1}}\right), 1-\left(1+\frac{p(2-\sqrt{3})}{p-1}\right)^{1-p}\right\} .
\end{aligned}
$$

El resultado de Xu and Roach [9] (véanse los Teoremas 7 y 8) establece que un espacio $X$ es uniformemente convexo si y solo si

$$
\|x+y\|^{p} \geq\|x\|^{p}+p\left\langle j_{p} x \mid y\right\rangle+\sigma_{\phi}(x, y)
$$

y $X$ es uniformemente suave si y solo si

$$
\|x+y\|^{p} \leq\|x\|^{p}+p\left\langle j_{p} x \mid y\right\rangle+\sigma_{\psi}(x, y)
$$

donde $j_{p} x$ es un elemento de $J_{p} x$ con $J_{p}: X \rightarrow 2^{X^{*}}$ y

$$
J_{p} x=\left\{x^{*} \in X^{*}:\left\langle x^{*} \mid x\right\rangle=\|x\|\left\|x^{*}\right\|,\left\|x^{*}\right\|=\|x\|^{p-1}\right\}
$$

un mapeo de dualidad de peso $t^{p-1}$.

En (1) la función $\phi$ es estríctamente creciente, convexa, tal que $\phi(0)=0$ y existe una constante $K>0$ tal que $\phi(t) \geq K \delta_{X}(t / 2)$. En (2) la función $\psi$ es estríctamente decreciente, convexa, tal que $\psi(0)=0$ y existe una constante $K>0$ tal que $\psi(t) \leq K \rho_{X}(t)$. Aquí

$$
\rho_{X}(\tau)=\sup \left\{\frac{\|x+y\|+\|x-y\|}{2}-1:\|x\|=1,\|y\|=\tau\right\}
$$

es el módulo de suavidad de $X$. En ambos (1) y (2)

$$
\sigma_{f}(x, y)=\int_{0}^{1} \frac{\max \{\|x+t y\|,\|x\|) p\}}{t} f\left(\frac{t\|y\|}{\max \{\|x+t y\|,\|x\|\}}\right) d t .
$$




\section{Preliminares}

Aparte de los preliminares ya presentados en la Introducción, vamos a necesitar algunos resultados y definiciones que presentamos a continuación. Referencias clásicas para la teoría de geometría en espacios de Banach son [2] y [6]. Este trabajo en particular ha sido inspirado por [3].

\subsection{Una caracterización de espacios uniformemente convexos}

La siguiente caracterización de espacios uniformemente cnvexos utiliza el mapeo normalizado de dualidad. La prueba la tomamos de [8].

Teorema 3. Sea $X$ un espacio de Banach y para $0<\varepsilon \leq 2$ defina

$$
\gamma(\varepsilon)=\inf \{1-\langle j x \mid y\rangle:\|x\|=\|y\|=1,\|x-y\| \geq \varepsilon, j x \in J x\} .
$$

Entonces $X$ es uniformemente convexo si y solo si $\gamma(\varepsilon)$ es positivo.

Prueba: Si $X$ es uniformemente convexo, para $\|x\|=\|y\|=1,\|x-y\| \geq \varepsilon$ y $j x$ en $J x$, se tiene que $\|(x+y) / 2\| \leq 1-\delta_{X}(\varepsilon)$ y

$$
\begin{array}{r}
\frac{1}{2}+\frac{1}{2}\langle j x \mid y\rangle=\frac{1}{2}\left\langle j x \mid \frac{x+y}{2}\right\rangle-\frac{1}{2}\langle j x \mid y\rangle \leq\|j x\| \| \\
=\frac{\|x+y\|}{2} \leq 1-\delta_{X}(\varepsilon)
\end{array}
$$

lo cual implica que $2 \delta_{X}(\varepsilon) \leq 1\langle j x \mid y\rangle$ y al tomar el ínfimo se tiene el resultado.

Para el recíproco tomamos $z=(x+y) /\|x+y\|$ para $x+y \neq 0$ y $\|x\|=\|y\|=1$, $\|x-y\| \geq \varepsilon$. Si $\|x+y\|<2-\varepsilon / 2$ entonces $1-\|x+y\| / 2>\varepsilon / 4$. Si $\|x+y\| \geq 2-\varepsilon / 2$ entonces

$$
\begin{aligned}
\|x-z\| & =\frac{\|x-y+x(\|x+y\|-2)\|}{\|x+y\|} \geq \frac{\|x-y\|-\|x\|(2-\|x+y\|)}{\|x+y\|} \\
& \geq \frac{\varepsilon-2+\|x+y\|}{2} \geq \frac{\varepsilon-\varepsilon / 2}{2}=\frac{\varepsilon}{4} .
\end{aligned}
$$

En forma similar $\|y-z\| \geq \varepsilon / 4$ y así

$$
\begin{aligned}
1-\frac{\|x+y\|}{2} & =\frac{1}{2}\left(2-\|x+y\|\left\langle j z \mid \frac{x+y}{\|x+y\|}\right\rangle\right)=\frac{1}{2}(2-\langle j z \mid x+y\rangle) \\
& =\frac{1}{2}(1-\langle j z \mid x\rangle+1-\langle j z \mid y\rangle) \geq \gamma\left(\frac{\varepsilon}{4}\right) .
\end{aligned}
$$

Por lo tanto

$$
1-\frac{\|x+y\|}{2} \geq \min \left\{\frac{\varepsilon}{4}, \gamma\left(\frac{\varepsilon}{4}\right)\right\} .
$$

Se concluye que $X$ es uniformemente convexo al tomar el ínfimo. 


\subsection{Subdiferenciales}

Una función $f: X \rightarrow \mathbb{R}$ se dice ser subdiferenciable en $x \in X$ si existe $x^{*}$ en $X^{*}$, llamado un subgradiante de $f$ en $x$, tal que $\left\langle x^{*} \mid y-x\right\rangle \leq f(y)-f(x)$ para todo $y$ en $X$. El conjunto de todos los subgradiantes de $f$ en $x$ se denota $\partial f(x)$, el mapa $\partial f: X \rightarrow 2^{X^{*}}$ se llama el sub-diferencial de $f$. No es difícil mostrar que para todo $x$ diferente de cero en un espacio de Banach $X$ se tiene $\partial\|x\|=\left\{x^{*} \in X^{*}:\left\langle x^{*} \mid x\right\rangle=\|x\|,\left\|x^{*}\right\|=1\right\}$. El siguiente resultado establece la relación entre $J_{p}$ y $\partial$.

Proposición 1. Para todo $x$ en un espacio de Banach $X$ se tiene $J_{p} x=\partial g(\|x\|)$ donde $g(t)=t^{p} / p$.

Prueba:

Usaremos la diferenciabilidad de $g$ y la convexidad de $g^{\prime}(t)=t^{p-1}$. Sean $x^{*}$ en $J_{p} x$ y $y$ en $X$. Si $\|y\|>\|x\|$ se tiene

$$
\left\|x^{*}\right\|=\|x\|^{p-1}=g^{\prime}(\|x\|) \leq \frac{g(\|y\|)-g(\|x\|)}{\|y\|-\|x\|},
$$

lo cual implica que $\left\langle x^{*} \mid y-x\right\rangle \leq\left\|x^{*}\right\|(\|y\|-\|x\|) \leq g(\|y\|)-g(\|x\|)$. En forma similar si $\|y\|<\|x\|$ se tiene $\left\langle x^{*} \mid y-x\right\rangle \leq g(\|y\|)-g(\|x\|)$.

Si $\|y\|=\|x\|$ entonces $\left\langle x^{*} \mid y-x\right\rangle \leq\left\|x^{*}\right\|(\|y\|-\|x\|)=0=g(\|y\|)-g(\|x\|)$. Por lo cual concluimos que para todo $y$ en $X$ tenemos $\left\langle x^{*} \mid y-x\right\rangle \leq g(\|y\|)-g(\|x\|)$ y así

$$
x^{*} \in \partial h(\|x\|)=\left\{x^{*} \in X^{*}:\left\langle x^{*} \mid y-x\right\rangle \leq g(\|y\|)-g(\|x\|), \forall y \in X\right\}
$$

donde pusimos $h(t)=t^{p-1}$. Hemos concluido que $J_{p} x \subset \partial g(\|x\|)$.

Recíprocamente considere $x^{*}$ en $\partial g(\|x\|)$ con $x$ diferente de cero.

$$
\begin{aligned}
& \left\|x ^ { * } \left|\||| x\|=\sup \left\{\left\langle x^{*} \mid y\right\rangle\|x\|:\|y\|=1\right\}=\sup \left\{\left\langle x^{*} \mid y\right\rangle:\|y\|=\|x\|\right\}\right.\right. \\
& \leq \sup \left\{\left\langle x^{*} \mid x\right\rangle+g(\|y\|)-g(\|x\|):\|y\|=\|x\|\right\}=\left\langle x^{*} \mid x\right\rangle \leq\left\|x^{*}\right\|\|x\|,
\end{aligned}
$$

lo cual implica que $\left\langle x^{*} \mid x\right\rangle=\left\|x^{*}\left|\||| x\|\right.\right.$. Ahora deseamos probar que $\left\|x^{*}\right\|=\|x\|^{p-1}=$ $g^{\prime}(\|x\|)$.

Si $t>\|x\|>0$ entonces

$$
\begin{aligned}
\left\|x^{*}\right\|(t-\|x\|) & =t\left\|x^{*}\right\|-\|x\|\left\|x^{*}\right\|=\left\langle x^{*} \mid x\right\rangle\left(\frac{t}{\|x\|}-1\right) \\
& =\left\langle x^{*} \mid \frac{x t}{\|x\|}-x\right\rangle \leq g(t)-g(\|x\|),
\end{aligned}
$$

lo cual implica que $(t-\|x\|)\left\|x^{*}\right\| \leq g(t) g(\|x\|)$. De forma similar, con $0<t<\|x\|$ tenemos $(t-\|x\|)\left\|x^{*}\right\| \leq g(t)-g(\|x\|)$. Haciendo $t \rightarrow\|x\|$ tenemos para $x \neq 0,\left\|x^{*}\right\|=$ $g^{\prime}(\|x\|)=\|x\|^{p-1}$.

En el caso $x=0, \partial g(0)=\left\{x^{*} \in X^{*}:\left\langle x^{*} \mid x\right\rangle \leq g(\|y\|), \forall y \in X\right\}$ y debemos probar que $\partial g(0)=\{0\}$. Como

$$
\left\langle x^{*} \mid y\right\rangle \leq g(\|y\|)=\int_{0}^{\|y\|} t^{p-1} d t \leq\|y\|^{p-1}\|y\|=\|y\|^{p},
$$

concluimos que $\left\|x^{*}\right\| \leq\|y\|^{p-1}$ para todo $y$ en $X$. Así $x^{*}=0$ y se tiene el resultado. 


\subsection{Espacios suaves}

Un hecho importante es la caracterización de aquellos espacios para los cuales el mapeo de dualidad posee un solo valor. Para nuestros própositos la Proposición 1 es suficiente pero no es mucho más difícil probar que para todo $x$ en un espacio de Banach $X$ se tiene $J_{h} x=\partial g(\|x\|)$ donde $g(t)=\int_{0}^{t} h(s) d s$. Aquí, como es usual $J_{h} x=\left\{x^{*} \in X^{*}:\left\langle x^{*} \mid x\right\rangle=\right.$ $\left.\|x\|\left\|x^{*}\right\|,\left\|x^{*}\right\|=h(\|x\|)\right\}$. De este modo $J_{h}$ posee un solo valor si y solo si existe un único $x^{*}$ en $X^{*}$ tal que $\left\langle x^{*} \mid x\right\rangle=\left\|x^{*}\left|\||| x\|\right.\right.$ y $\left\|x^{*}\right\|=h(\|x\|)$ si y solo si existe un único $x^{*}$ en $X^{*}$ tal que $\left\|x^{*}\right\|=1$ y $\left\langle x^{*} \mid x\right\rangle=\|x\|$. Es decir, $\partial\|x\|$ posee un solo elemento donde $\partial\|x\|=\left\{x^{*} \in X^{*}:\left\langle x^{*} \mid x\right\rangle=\|x\|,\left\|x^{*}\right\|=1\right\}$.

Por definición, un espacio de Banach $X$ se dice ser suave si para todo $x \neq 0, \partial\|x\|$ posee un solo elemento. El siguiente resultado es una consecuencia sencilla del párrafo anterior.

Lema 1. Un espacio de Banach es suave si y solo si todo mapeo de dualidad $J_{h}$ de función de peso h posee un solo valor.

Otra caracterización que utilizaremos de espacios de Banach suaves está dada por el siguiente teorema.

Teorema 4. Un espacio de Banach $X$ es suave si y solo si la norma es Gâteaux diferenciable sobre $X \backslash\{0\}$.

La prueba se puede leer en [6]. Recordemos que para espacios de Banach $X, Y$ una función $F: D \rightarrow Y$ con $D$ un subconjuno abierto de $X$ se dice ser Gâteaux-diferenciable en $x$ si existe un operador lineal $F^{\prime}(x): X \rightarrow Y$ tal que para todo $y$ en $X$

$$
\lim _{t \rightarrow 0} \frac{F(x+t y)-F(x)}{t}=F^{\prime}(x) y .
$$

\subsection{Dualidad entre espacios uniformemente convexos y uniformemente suaves}

Un espacio normado $X$ se dice ser uniformemente suave siempre que dado $\varepsilon>0$ existe $\delta>0$ tal que si $\|x\|=1$ y $\|y\|<\delta$ entonces $\|x+y\|+\|x-y\|<2+\varepsilon\|y\|$.

En general para un espacio de Banach $X$

$$
\limsup _{t \rightarrow 0} \frac{\rho_{X}(2 t)}{\rho_{X}(t)} \leq 4
$$

y existe una constante $c>0$ tal que para todo $0<s<t$ se tiene

$$
\frac{\rho_{X}(t)}{t^{2}} \leq c \frac{\rho_{X}(s)}{s^{2}}
$$

Así como el módulo de convexidad caracteriza los espacios uniformemente convexos, el módulo de suavidad caracteriza los espacios uniformemente suaves. 
Teorema 5. Un espacio normado $X$ es uniformemente suave si y solo si

$$
\lim _{t \rightarrow 0^{+}} \frac{\rho_{X}(t)}{t}=0
$$

No es difícil demostrar que el siguiente resultado.

Proposición 2. Todo espacio de Banach unifomemente suave es suave.

Es usual en matemáticas el estudio de conceptos en cercana relación de modo que las características de uno se reflejan en el otro. Este tipo de "dualidad" es central en la geometría de espacios de Banach. El enlace fundamental entre las nociones de uniformemente convexos y uniformemente suaves son las fórmulas de dualidad de Lindenstrauss [7].

Proposición 3. Sea $X$ un espaco de Banach, para todo $\tau>0$, $x$ en $X$ con $\|x\|=1$ y $x^{*}$ en $X^{*}$ con $\left\|x^{*}\right\|=1$ se tiene

$$
\begin{aligned}
& \rho_{X^{*}}=\sup \left\{\frac{\tau \varepsilon}{2}-\delta_{X}(e): 0<\varepsilon \leq 2\right\}, \\
& \rho_{X}=\sup \left\{\frac{\tau \varepsilon}{2}-\delta_{X^{*}}(e): 0<\varepsilon \leq 2\right\} .
\end{aligned}
$$

En particular usaremos el corolario de este esultado.

Corolario 1. Para todo espacio de Banach $X$ la función $\rho_{X}(t) / t$ es no-decreciente $y$ $\rho_{x}(t) \leq t$

El siguiente resultado da la dualidad entre espacios uniformemente convexos y uniformmente suaves.

Teorema 6. Sea $X$ un espacio de Banach.

a. $X$ es uniformemente suave si $y$ solo si $X^{*}$ es uniformemente convexo.

b. $X$ es uniformemente convexo si y solo si $X^{*}$ es uniformemente suave.

Prueba: Para " $a$. $\rightarrow$ " si $X^{*}$ no es uniformemente convexo, existe $\left.\left.\varepsilon_{0} \in\right] 0,2\right]$ con $\delta_{X^{*}}\left(\varepsilon_{0}\right)=0$. Por la segunda fórmula en Proposición 3 obtenemos que para todo $\tau>0$,

$$
0<\frac{\varepsilon_{0}}{2} \leq \frac{\rho_{X}(\tau)}{\tau}
$$

Lo cual significa que $X$ no es uniformemente suave.

Para " $a . \leftarrow$ " asuma que $X$ no es uniformemente suave. Entonces

$$
\lim _{t \rightarrow 0^{+}} \frac{\rho_{X}(t)}{t} \neq 0
$$

lo cual significa que existe $\varepsilon>0$ tal que para todo $\delta>0$ podemos encontrar $t_{\delta}$ con $0<t_{\delta}<\delta$ y $t_{\delta} \varepsilon \leq \rho_{X}\left(t_{\delta}\right)$. De este modo existe una sucesión $\left(\tau_{n}\right)_{n}$ tal que $0<\tau_{n}<1$, 
$\tau_{n} \rightarrow 0 \mathrm{y} \rho_{X}\left(\tau_{n}\right)>\varepsilon \tau_{n} / 2$. Por la segunda fórmula de la Proposición 3 para todo $n$ existe $\varepsilon_{n}$ en $\left.] 0,2\right]$ tal que

$$
\frac{\varepsilon}{2} \tau_{n} \leq \frac{\tau_{n} \varepsilon_{n}}{2}-\delta_{X^{*}}\left(\varepsilon_{n}\right) \quad \text { lo cual implica } \quad 0<\delta_{X^{*}}\left(\varepsilon_{n}\right) \leq \frac{\tau_{n}}{2}\left(\varepsilon_{n}-\varepsilon\right),
$$

en particular $\varepsilon<\varepsilon_{n}$ y $\delta_{X^{*}}\left(\varepsilon_{n}\right) \rightarrow 0$. Dado que $\delta_{X^{*}}$ es una función no decreciente tenemos $\delta_{X^{*}}(\varepsilon) \leq \delta_{X^{*}}\left(\varepsilon_{n}\right) \rightarrow 0$. Así $X^{*}$ no es uniformemente convexo.

Para obtener $b$. basta intercambiar los papeles de $X$ y $X^{*}$ en la prueba y usar la primera fórmula en Proposición 3.

Por el Teorema de Milman-Pettis, todo espacio uniformemente convexo es reflexivo así por el resultado anterior si $X$ es un espacio de Banach uniformemente suave ambos $X$ y $X^{*}$ son reflexivos. Por el Teorema de Hahn-Banach si $X$ es reflexivo entonces todo mapeo de dualidad $J_{h}$ es sobreyectivo y en particular $J_{p}$ y $J_{q}^{*}$ son sobreyectivos donde hemos tomado $p \in] 0, \infty\left[, 1 / p+1 / q=1\right.$ y $J_{q}^{*}$ el mapeo de dualidad $X^{*} \rightarrow X$ con función de peso $t \mapsto t^{q-1}$. dado que $X$ es uniformemente suave, por la Proposición 2 y el Lema 1 tenemos que $J_{p}$ posee un solo valor y así el inverso $J_{p}^{-1}$ de $J_{p}$ está dado por $J_{p}^{-1} x^{*}=\left\{x \in X: j_{p} x=x^{*}\right\}$ donde $j_{p} x$ denota el único elemento en $J_{p} x$. No es difícil verificar que si $J_{h^{-1}}^{*}$ denota el mapeo de dualidad sobre $X^{*}$ con función de peso $h^{-1}$ entonces $x^{*} \in J_{h} x$ si y solo si $x \in J_{h^{-1}}^{*}\left(x^{*}\right)$. Ahora como $\left(t \mapsto t^{p-1}\right)^{-1}=\left(t \mapsto t^{q-1}\right)$ tenemos que $x$ está en $J_{q}^{*} x^{*}$ si y solo si $x^{*}$ está en $J_{p} x=\left\{j_{p} x\right\}$ si y solo si $x$ está en $J_{p}^{-1} x^{*}$. De este modo se concluye el siguiente resultado.

Proposición 4. Para todo espacio de Banach $X$ uniformemente suave se tiene $J_{p}^{-1}=J_{q}^{*}$.

\subsection{Un par de conjuntos útiles y sus propiedades}

Para un espacio de Banach $X$, defina los conjuntos

$$
\begin{array}{r}
A=\left\{\phi: \mathbb{R}^{+} \rightarrow \mathbb{R}^{+}: \phi(0)=0, \phi\right. \text { es estríctamente creciente, } \\
\text { convexa y existe } \left.K>0 \text { con } K \delta_{X}(t / 2) \leq \phi(t)\right\}
\end{array}
$$

y

$$
\begin{array}{r}
B=\left\{\phi: \mathbb{R}^{+} \rightarrow \mathbb{R}^{+}: \phi(0)=0, \phi\right. \text { es no decreciente } \\
\text { convexa y existe } \left.K>0 \text { con } K \rho_{X}(t / 2) \geq \phi(t)\right\}
\end{array}
$$

Sea $p$ un número en el intervalo $] 1, \infty\left[\right.$ y sea $J_{p}$ el mapeo de dualidad sobre $X$ con función de peso $\phi_{p}(t)=t^{p-1}$.

Lema 2. Si para todo $x, y$ en $X \backslash\{0\}$ con $\max \{\|x\|, \mid y \|\}=1$ y para todo $\phi$ en $A$ se tiene $\phi(\|x-y\|) \leq\left\langle j_{p} x-j_{p} y \mid x-y\right\rangle$ entonces

$$
(\max \{\|x\|,\|y\|\})^{p} \phi\left(\frac{\|x-y\|}{\max \{\|x\|,\|y\|\}}\right) \leq\left\langle j_{p} x-j_{p} y \mid x-y\right\rangle .
$$


Prueba: Podemos asumir que $\|y\| \leq\|x\|$ y dado que $\max \left\{\left\|\frac{x}{\|x\|}\right\|,\left\|\frac{y}{\|y\|}\right\|\right\}=1$ tenemos

$$
\phi\left(\frac{\|x-y\|}{\|x\|}\right) \leq\left\langle j_{p}\left(\frac{x}{\|x\|}\right)-j_{p}\left(\frac{y}{\|x\|}\right) \mid \frac{x-y}{\|x\|}\right\rangle .
$$

Basta mostrar que

$$
\phi\left(\frac{\|x-y\|}{\|x\|}\right) \leq \frac{1}{\|x\|^{p}}\left\langle j_{p} x-j_{p} y \mid x-y\right\rangle
$$

de lo cual sigue el resultado. De hecho basta tomar $\lambda=1 /\|x\|$ en

$$
J_{p}(\lambda x)=\operatorname{sign}(\lambda)(|\lambda|)^{p-1} J_{p}(x)
$$

Para probar (4) primero note que $J_{p}(-x)=-J_{p} x$. Ahora asuma $\lambda>0$ y $\|x\| \neq 0$. Para $\alpha=\lambda^{p-1}$ y $x^{*}$ en $J_{p} x$ tenemos $\left\langle\alpha x^{*} \mid \lambda x\right\rangle=\alpha \lambda\left\langle x^{*} \mid x\right\rangle=\alpha \lambda\left\|x\left|\left\|\left|x^{*}\|=\| \lambda x\right|\right\|\right| \mid a x^{*}\right\| \mathrm{y}$ $\left\|\alpha x^{*}\right\|=\alpha\left\|x^{*}\right\|=\lambda^{p-1}\|x\|^{p-1}$. Así $\alpha J_{p} x \subset J_{p}(\lambda x)$.

Para $x^{*}$ en $J_{p}(\lambda x)$ tenemos

$$
\left\langle\frac{x^{*}}{\alpha} \mid x\right\rangle=\frac{1}{\alpha \lambda}\left\langle x^{*} \mid x\right\rangle=\frac{\|\lambda x\|\left\|x^{*}\right\|}{\alpha \lambda}=\|x\|\left\|\frac{x^{*}}{\alpha}\right\|
$$

y

$$
\left\|\frac{x^{*}}{\alpha}\right\|=\frac{\|x\|^{*}}{\alpha}=\frac{(\lambda\|x\|)^{p-1}}{\alpha}=\|x\|^{p-1} .
$$

Así $\alpha^{-1} J_{p}(\lambda x) \subset J_{p} x$ lo cual demuestra (4).

Lema 3. Para todo $\phi$ en $A$, para todo $x, y$ diferente de cero en $X$ y para todo $p$ en $(1, \infty)$ la función

$$
S(t)=\frac{(\max \{\|x+t y\|,\|x\|\})^{p}}{t} \phi\left(\frac{t\|y\|}{\max \{\|x+t y\|,\|x\|\}}\right)
$$

es integrable en el sentido de Riemann en [0,1].

Prueba: Note que $t \mapsto \| x+t y||$ es continua dado que ||$\left|x+t y\|-\| x_{s} y \|\right||\leq||y||| t-s \mid$ y así $t \mapsto \max \{\|x+t y\|,\|y\|\}$ es una función continua de $t$. Dado que $\phi$ es continua y convexa, para $t$ en $[0,1]$ tenemos

$$
\phi\left(\frac{t\|y\|}{\max \{\|x+t y\|,\|x\|\}}\right) \leq \phi\left(\frac{t\|y\|}{\|x\|}\right) \leq t \phi\left(\frac{\|y\|}{\|x\|}\right),
$$

lo cual implica que

$$
0 \leq \int_{0}^{1} S(t) d t \leq \int_{0}^{1}(\max \{\|x+t y\|,\|x\|\})^{p} \phi\left(\frac{\|y\|}{\|x\|}\right) d t
$$

de lo cual sigue el resultado. 


\section{Caracterización mediante desigualdades}

Esta sección presentamos la caracterización de espacios de Banach uniformemente convexos de [9] mediante una desigualdad que involucra funciones estríctamente decrecientes [3]. Luego de esto, usando el mapeo de dualidad, presentamos el resultado dual [9] para espacios de Banach uniformemente suaves.

\subsection{El caso de espacios uniformemente convexos}

Teorema 7. Sea $X$ un espacio de Banach, para cualquier $p$ en $] 1, \infty[y$ para todo $x, y$ en $X$ son equivalentes:

a. X es uniformemente convexo.

b. Existe una función $\phi_{p}$ en A tal que

$$
(\max \{\|x\|,\|y\|\})^{p} \phi_{p}\left(\frac{\|x-y\|}{\max \{\|x\|,\|y\|\}}\right) \leq\left\langle j_{p} x-j_{p} y \mid x-y\right\rangle .
$$

c. Existe una función $\phi_{p}$ en A tal que

$$
\|x\|^{p}+p\left\langle j_{p} x \mid y\right\rangle+\sigma_{p}(x, y) \leq\|x+y\|^{p} .
$$

Prueba: Para probar $a$. $\mapsto b$. por el Lema 2 es suficiente mostrar que si se tiene $\max \{\|x\|,\|y\|\}=1$ entonces $\phi_{p}(\|x-y\|) \leq\left\langle j_{p} x-j_{p} y \mid x-y\right\rangle$. Por el Teorema 2 tenemos

$$
\|\lambda x+(1-\lambda) y\|^{p}+s(p, \lambda,\|x-y\|) \leq \lambda\|x\|^{p}+(1-\lambda)\|y\|^{p}
$$

y en particular

$$
\begin{gathered}
\|x+(1-\lambda)(y-x)\|^{p}-\|x\|^{p} \leq(1-\lambda)\left(\|x\|^{p}-\|y\|^{p}\right)-s(p, \lambda,\|x-y\|), \\
\|y+\lambda(x-y)\|^{p}-\|y\|^{p} \leq \lambda\left(\|x\|^{p}-\|y\|^{p}\right)-s(p, \lambda,\|x-y\|) .
\end{gathered}
$$

Por la Proposición 1, para todo $x$ en $X$

$$
J_{p} x=\partial \int_{0}^{\|x\|} t^{p-1} d t=\partial\left(\frac{\|x\|^{p}}{p}\right)=\left\{x^{*} \in X^{*}:\left\langle X^{*} \mid Y-X\right\rangle \leq \frac{\|y\|^{p}-\|x\|^{p}}{p}\right\},
$$

así

$$
\begin{aligned}
p\left\langle j_{p} x \mid y-x\right\rangle & =\frac{p}{1-\lambda}\left\langle j_{p} x \mid x+(1-\lambda)(y-x)-x\right\rangle \\
& \leq \frac{1}{1-\lambda}\left(\|x+(1-\lambda)(y-x)\|^{p}-\|x\|^{p}\right) \leq\|y\|^{p}-\|x\|^{p}-\frac{s(p, \lambda,\|x-y\|}{1-\lambda} \\
& \leq\|y\|^{p}-\|x\|^{p}-\lim \sup _{\lambda \rightarrow 1} \frac{s(p, \lambda,\|x-y\|}{1-\lambda}
\end{aligned}
$$


y

$$
\begin{aligned}
p\left\langle j_{p} y \mid x-y\right\rangle & =\frac{p}{\lambda}\left\langle j_{p} y \mid y+\lambda(x-y)-y\right\rangle \\
& \leq \frac{1}{\lambda}\left(\|y+\lambda(x-y)\|^{p}-\|y\|^{p}\right) \leq\|x\|^{p}-\|y\|^{p}-\frac{s(p, \lambda,\|x-y\|}{\lambda} \\
& \leq\|x\|^{p}-\|y\|^{p}-\lim \sup _{\lambda \rightarrow 0} \frac{s(p, \lambda,\|x-y\|}{1-\lambda} .
\end{aligned}
$$

De la segunda parte del Teorema 2 y estas dos desigualdades obtenemos que

$$
\left\langle j_{p} x-j_{p} y \mid x-y\right\rangle \geq \frac{K_{p}}{p} \delta_{X}\left(\frac{\|x-y\|}{2}\right) .
$$

b. sigue de tomar $\phi_{p}(t)=\delta_{X}(t / 2)$ el cual está en $A$ por el Teorema 1 y dado que en un espacio uniformemente convexo el módulo de convexidad es una función estríctamente creciente [3].

Para probar $b . \mapsto c$. tomamos $\Phi(t)=\|x+t y\|^{p} / p$ y $0=t_{0}<t_{1}<\cdots<t_{n}=1$ una partición arbitraria del intervalo [0,1]. Entonces

$$
\begin{aligned}
\frac{1}{p}\|x+y\|^{p}-\frac{1}{p}\|x\|^{p} & =\Phi(1)-\Phi(0)=\sum_{k=0}^{n-1} \Phi\left(t_{k+1}\right)-\Phi\left(t_{k}\right) \\
& =\sum_{k=0}^{n-1} \frac{\left\|x+t_{k+1} y\right\|^{p}-\left\|x+t_{k} y\right\|^{p}}{p}
\end{aligned}
$$

y por la Proposición 1

$$
\frac{1}{p}\|x+y\|^{p}-\frac{1}{p}\|x\| \geq \sum_{k=0}^{n-1}\left\langle j_{p}\left(x+t_{k} y\right) \mid y\right\rangle\left(t_{k+1}-t_{k}\right)
$$

lo cual implica

$$
\begin{aligned}
& \|x+y\|^{p}-\|x\|^{p}-p\left\langle j_{p} x \mid y\right\rangle \geq p \sum_{k=0}^{n-1} \frac{\left\langle j_{p}\left(x+t_{k} y\right)-j_{p} x \mid t_{k} y\right\rangle}{t_{k}} \\
& \geq p \sum_{k=0}^{n-1} \frac{\left(\max \left\{\left\|x+t_{k} y\right\|,\|x\|\right\}\right)^{p}}{t_{k}} \phi_{p}\left(\frac{\left\|t_{k} y\right\|}{\max \left\{\left\|x+t_{k} y\right\|,\|x\|\right\}}\right)\left(t_{k+1}-t_{k}\right) .
\end{aligned}
$$

Usando el Lema 3, c. sigue de la desigualdad

$$
\|x+y\|^{p}=\|x\|^{p}-p\left\langle j_{p} x \mid y\right\rangle \geq \lim _{n \rightarrow \infty} p \sum_{k=1}^{n-1}\left(t_{k+1}-t_{k}\right) S\left(t_{k}\right)=p \int_{0}^{1} S(t) d t .
$$


Para probar $c . \mapsto a$. note que con $p=2, j_{p}$ es precisamente el mapeo normalizado de dualidad. Así para cualesquiera $x, y$ en $X$ con $\|x\|=\|y\|=1$ se tiene

$$
\begin{aligned}
& 0=\|y\|^{2}-\|x\|^{2}=\|x+(y-x)\|^{2}-\|x\|^{2} \\
& 2\left\langle j_{2} x \mid y-x\right\rangle+2 \int_{0}^{1} \frac{(\max \{\|x+t(y-x)\|, 1\})^{2}}{t} \phi_{2}\left(\frac{t\|y-x\|}{\|x+t(y-x)\|}\right) d t \\
& \quad=2\left\langle j_{2} x \mid y-x\right\rangle+2 \int_{0}^{1} \frac{\phi_{2}(t|| x-y \|)}{t}=2\left\langle j_{2} x \mid y-x\right\rangle+2 \int_{0}^{\|x-y\|} \frac{\phi_{2}(t)}{t}
\end{aligned}
$$

lo cual implica

$$
1-\left\langle j_{2} x \mid y\right\rangle=\left\langle j_{2} x \mid x\right\rangle-\left\langle j_{2} x \mid y\right\rangle \geq \int_{0}^{\|x-y\|} \frac{\phi_{2}(t)}{t} d t .
$$

Como conclusión se obtiene que

$$
1-\left\langle j_{2} x \mid y\right\rangle \geq \int_{0}^{\varepsilon} \frac{\phi_{2}(t)}{t} d t
$$

para todo $\|x\|=\|y\|=1$ y $\|x-y\| \geq \varepsilon$. Que $X$ es uniformemente convexo sigue del Teorema 3 .

\subsection{El caso de espacios uniformemente suaves}

En esta sección para $p$ en $(1, \infty)$ consideramos $q$ tal que $1 / p+1 / q=1$. Si $X$ es un espacio de Banach uniformamente suave se tiene que $X^{*}$ es uniformemente convexo por el Teorema 6. Por la Proposición 2 y por el Lema 1 se tiene que $J_{p}$ posee un solo valor. Por el Teorema $7 a$. $\mapsto b$. tenemos que para todo $x^{*}, y^{*}$ en $X^{*}$ y $K_{q}$ dado en la segunda parte del Teorema 2

$$
\begin{aligned}
\left\|j_{q}^{*} x^{*}-j_{q}^{*} y^{*}||\right\| x^{*}-y^{*}|| & \geq\left\langle j_{q}^{*} x^{*}-j_{q}^{*} y^{*} \mid x^{*}-y^{*}\right\rangle \geq \\
& \frac{K_{q}}{q}\left(\max \left\{\left\|x^{*}\right\|,\left\|y^{*}\right\|\right\}^{q} \delta_{X^{*}}\left(\frac{\left\|x^{*}-y^{*}\right\|}{2 \max \left\{\left\|x^{*}\right\|,\left\|y^{*}\right\|\right\}}\right)\right.
\end{aligned}
$$

lo cual implica que para $\hat{\delta}_{X^{*}}(t)=\delta_{X^{*}}(t) / t$

$$
\left\|j_{q}^{*} x^{*}-j_{q}^{*} y^{*}\right\| \geq \frac{K_{q}}{2 q}\left(\max \left\{\left\|x^{*}\right\|,\left\|y^{*}\right\|\right\}^{q-1} \hat{\delta}_{X^{*}}\left(\frac{\left\|x^{*}-y^{*}\right\|}{2 \max \left\{\left\|x^{*}\right\|,\left\|y^{*}\right\|\right\}}\right) .\right.
$$

Por la Proposición 4 podemos tomar $x^{*}=j_{p} x$ en $J_{p} x$ y $y^{*}=j_{p} y$ en $J_{p} y$ para obtener

$$
\frac{2 q\|x-y\|}{K_{q}(\max \{\|x\|,\|y\|\})} \geq \hat{\delta}_{X^{*}}\left(\frac{\left\|j_{p} x-j_{p} y\right\|}{2(\max \{\|x\|,\|y\|\})^{p-1}}\right) .
$$

Dado que por el Corolario 1 la función $4 \hat{\rho}_{X}(t)=\rho_{X}(t) / t$ es no decreciente, tenemos que para todo $x$ distinto de $y$, con $j_{p} x$ distinto de $j_{p} y$

$$
\begin{aligned}
\hat{\rho}_{X}\left(\frac{8 q\|x-y\|}{K_{q}(\max \{\|x\|,\|y\|\})}\right) & \geq \hat{\rho}_{X}\left(4 \hat{\delta}_{X^{*}}\left(\frac{\| j_{p} x-j_{p} y}{2(\max \{\|x\|,\|y\|\})^{p-1}}\right)\right) \\
& \geq \frac{\left\|j_{p} x-j_{p} y\right\|}{8(\max \{\|x\|,\|y\|\})^{p-1}}
\end{aligned}
$$


donde hemos hecho uso de las propiedades del módulo de suavidad. Por la Proposición 3 tenemos para todo $\varepsilon$ en $] 0,2]$

$$
\rho_{X}\left(\frac{4 \delta_{X^{*}}(\varepsilon)}{\varepsilon}\right) \geq 2 \delta_{X^{*}}(\varepsilon)-\delta_{X^{*}}(\varepsilon)=\delta_{X^{*}}(\varepsilon)
$$

y así $\hat{\rho}_{X}\left(4 \hat{\delta}_{X^{*}}(\varepsilon)\right) \geq \varepsilon / 4$.

El siguiente resultado es el dual del Teorema 7 para espacios uniformemente suaves.

Teorema 8. Sea $X$ un espacio de Banach, para cualquier $p$ en $] 1, \infty[y$ para todo $x, y$ en $X$ son equivalentes:

a. X es uniformemente suave.

b. $J_{p}$ tiene un solo valor y existe una función $\phi_{p}$ en $B$ tal que para todo $x, y$ en $X$

$$
\left\|j_{p} x-j_{p} y\right\| \leq(\max \{\|x\|,\|y\|\})^{p-1} \hat{\phi}_{p}\left(\frac{\|x-y\|}{\max \{\|x\|,\|y\|\}}\right)
$$

donde $\hat{\phi}(t)=\phi(t) / t$.

c. Existe una función $\phi_{p}$ en $B$ tal que

$$
\|x+y\|^{p} \leq\|x\|^{p}+p\left\langle j_{p} x \mid y\right\rangle+\sigma_{p}(x, y) .
$$

Prueba: Para $a . \mapsto b$. notamos que en el párrafo anterior probamos la desigualdad

$$
\begin{aligned}
\left\|j_{p} x-j_{p} y\right\| & \leq 8(\max \{\|x\|,\|y\|\})^{p-1} \hat{\rho}_{X}\left(\frac{8 q\|x-y\|}{K_{q}(\max \{\|x\|,\|y\|\})}\right) \\
& =\frac{K_{q}(\max \{\|x\|,\|y\|\})^{p}}{q\|x-y\|} \rho_{X}\left(\frac{8 q\|x-y\|}{\max \{\|x\|,\|y\|\}}\right) .
\end{aligned}
$$

Dado que cualquier múltiplo positivo de el módulo de suavidad es evidentemente un elemento de $B$, queremos factorizar hasta desigualdad, el coeficiente $8 q / K_{q}$ en la última expresión. Para lograrlo consideramos dos casos.

Si $8 q \leq K_{q}$ por la convexidad de $\rho_{X}$ tenemos

$$
\begin{aligned}
\left\|j_{p} x-j_{p} y\right\| & \leq 8 \frac{(\max \{\|x\|,\|y\|\})^{p}}{\|x-y\|} \rho_{X}\left(\frac{\|x-y\|}{\max \{\|x\|,\|y\|\}}\right) \\
& =8(\max \{\|x\|,\|y\|\})^{p-1} \hat{\rho}_{X}\left(\frac{\|x-y\|}{\max \{\|x\|,\|y\|\}}\right) .
\end{aligned}
$$


Si $8 q>K_{q}$ por $(3)$

$$
\begin{aligned}
& \left\|j_{p} x-j_{p} y\right\| \\
& \leq \frac{8^{2} q}{K_{q}}(\max \{\|x\|,\|y\|\})^{p-2}\|x-y\| \times \\
& \times \rho_{X}\left(\frac{8 q\|x-y\|}{K_{q} \max \{\|x\|,\|y\|\}}\right)\left(\frac{8 q\|x-y\|}{K_{q} \max \{\|x\|,\|y\|\}}\right)^{-2} \\
& \leq c \frac{8^{2} q}{K_{q}}(\max \{\|x\|,\|y\|\})^{p-2}\|x-y\| \times \\
& \times \rho_{X}\left(\frac{\|x-y\|}{\max \{\|x\|,\|y\|\}}\right)\left(\frac{\|x-y\|}{\max \{\|x\|,\|y\|\}}\right)^{-2} \\
& =c \frac{8^{2} q}{K_{q}}(\max \{\|x\|,\|y\|\})^{p-1} \hat{\rho}_{X}\left(\frac{\|x-y\|}{\max \{\|x\|,\|y\|\}}\right) .
\end{aligned}
$$

El resultado sigue de tomar $\phi_{p}(t)=\max \left\{8,8^{2} c q \rho_{X}(t) / K_{q}\right\}$.

Para $b$. $\mapsto c$. dado que $J_{p}$ tiene un solo valor, por el Lema $1 X$ es suave y por el Teorema 4 la norma es Gâteaux diferenciable en $X \backslash\{0\}$. Así la función $\Phi(t)=\|x+t y\|^{p}$ para $t \in] 0,1]$ es continuamente diferenciable con derivada $\Phi^{\prime}(t)=p\|x+t y\|^{p-1} \frac{d}{d t}(\|x+t y\|)$. Dado que

$$
\left\langle j_{p}\left(\frac{x+t y}{\|x+t y\|}\right) \mid \frac{x+t y}{\|x+t y\|}\right\rangle=\frac{1}{\|x+t y\|^{p}}\left\langle j_{p}(x+t y) \mid x+t y\right\rangle=1,
$$

por la prueba de $b$. $\mapsto c$. de Teorema 4 tenemos que

$$
\begin{aligned}
\lim _{h \rightarrow 0} \frac{\left\|\frac{x+t y}{\|x+t y\|}+\frac{h y}{\|x+t y\|}\right\|-1}{h} & =\left\langle j_{p}\left(\frac{x+t y}{\|x+t y\|}\right) \mid \frac{y}{\|x+t y\|}\right\rangle \\
& =\frac{\left\langle j_{p}(x+t y) \mid y\right\rangle}{\|x+t y\|^{p}} .
\end{aligned}
$$

Así

$$
\|x+t y\|^{\prime}=\frac{\left\langle j_{p}(x+t y) \mid y\right\rangle}{\|x+t y\|^{p-1}}
$$

y entonces $\Phi^{\prime}(t)=p\left\langle j_{p}(x+t y) \mid y\right\rangle$.

Ahora

$$
\|x+y\|^{p}-\|x\|^{p}-\left\langle j_{p} x \mid y\right\rangle=\Phi(1)-\Phi(0)-\Phi^{\prime}(0)=p \int_{0}^{1}\left\langle j_{p}(x+t y)-j_{p} x \mid y\right\rangle d t
$$

y por hipótesis

$$
\begin{aligned}
& \|x+y\|^{p}-\|x\|^{p}-p\left\langle j_{p} x \mid y\right\rangle \\
& \leq p\|y\| \int_{0}^{1}(\max \{\|x \mid,\| y \|\})^{p-1} \hat{\phi}_{p}\left(\frac{\|t y\|}{\max \{\|x+t y\|,\|x\|\}}\right) d t \\
& =p \int_{0}^{1} \frac{(\max \{\|x+t y\|,\|x\|\})^{p}}{t} \phi_{p}\left(\frac{t\|x\|}{\max \{\|x+t y\|,\|x\|\}}\right) d t
\end{aligned}
$$


de lo cual sigue el resultado.

Para $c$. $\mapsto a$. sea $\|x\|=1$ y $\|y\| \leq t$. Por hipótesis tenemos que

$$
\begin{gathered}
\|x+y\| \leq\left(\|x\|^{p}+p\left\langle j_{p} x \mid y\right\rangle+\sigma_{p}(x, y)\right)^{\frac{1}{p}}=\left(1+p\left\langle j_{p} x \mid y\right\rangle+\sigma_{p}(x, y)\right)^{\frac{1}{p}}, \\
\|x-y\| \leq\left(\|x\|^{p}-p\left\langle j_{p} x \mid y\right\rangle+\sigma_{p}(x,-y)\right)^{\frac{1}{p}}=\left(1-p\left\langle j_{p} x \mid y\right\rangle+\sigma_{p}(x,-y)\right)^{\frac{1}{p}},
\end{gathered}
$$

lo cual implica que

$$
\|x+y\|+\|x-y\| \leq \alpha^{\frac{1}{p}}\left\{\left(1+\frac{p}{\alpha}\left\langle j_{p} x \mid y\right\rangle\right)^{\frac{1}{p}}+\left(1-\frac{p}{\alpha}\left\langle j_{p} x \mid y\right\rangle\right)^{\frac{1}{p}}\right\}
$$

donde $\alpha=1+\max \left\{\sigma_{p}(x, y), \sigma_{p}(x,-y)\right\}$. Dado que $\left|p \alpha^{-1}\left\langle j_{p} x \mid y\right\rangle\right| \leq p t$, para $t$ lo suficientemente pequeño tenemos

$$
\begin{aligned}
& \left(1+\frac{p}{\alpha}\left\langle j_{p} x \mid y\right\rangle\right)^{\frac{1}{p}}=\sum_{n=0}^{\infty}\left(\begin{array}{c}
1 / p \\
n
\end{array}\right)\left(\frac{p}{\alpha}\left\langle j_{p} x \mid y\right\rangle\right)^{n}, \\
& \left(1-\frac{p}{\alpha}\left\langle j_{p} x \mid y\right\rangle\right)^{\frac{1}{p}}=\sum_{n=0}^{\infty}\left(\begin{array}{c}
1 / p \\
n
\end{array}\right)\left(-\frac{p}{\alpha}\left\langle j_{p} x \mid y\right\rangle\right)^{n},
\end{aligned}
$$

donde $\left(\begin{array}{c}1 / p \\ n\end{array}\right)=\frac{(1 / p)(1 / p-1) \cdots(1 / p-n+1)}{n !}$. Como resultado, para $t$ lo suficientemente pequeño

$$
\|x+y\|+\|x-y\| \leq 2 \alpha^{\frac{1}{p}} \sum_{n=0}^{\infty}\left(\begin{array}{c}
1 / p \\
2 n
\end{array}\right)\left(-\frac{p}{\alpha}\left\langle j_{p} x \mid y\right\rangle\right)^{2 n} \leq 2 \alpha \leq 2(1 \sigma(t)),
$$

$\operatorname{con} \sigma(t)=\sup \left\{\sigma_{p}(x, y), \sigma_{p}(x,-y):\|x\|=1,\|y\| \leq t\right\}$.

Por la definición del módulo de suavidad de $X$ tenemos que $\rho_{X}(t) \leq \sigma(t)$ para todo $t>0$. De la expresión de $\sigma_{p}$ se sigue que $\lim _{t \rightarrow 0} \frac{\sigma(t)}{t}=0$ y entonces $\lim _{t \rightarrow 0} \frac{\rho_{X}(t)}{t}=0$. Es decir $X$ es uniformemente suave.

Históricamente diferentes autores han brindado diferentes definiciones para el módulo de convexidad. Uno de los primeros interesados en establecer las relaciones entre estas diferentes definiciones y sus consecuencias fué M. M. Day en [4, 5].

Las aplicaciones de la dualidad entre espacios de Banach uniformemente convexos y uniformemente suaves son varias. Entre ellas se encuentran las mejores aproximaciones, las proyecciones métricas y los conjuntos de Chebyshev, las sucesiones de operadores lineales de contracción, puntos fijos de mapeos de Lipschitz pseudocontractivos, y los operadores de aumentación.

\section{Reconocimientos}

Este trabajo es parte del trabajo de graduación [10] presentado por el autor para el Diploma en Matemáticas del Abdus Salam International Center for Theoretical Physics en el periodo académico 1997-1998 bajo la amable dirección del profesor C. E. Chidume a quien extiendo mi humilde gratitud. 


\section{Bibliografía}

[1] Beurling, A.; Livingston, A. E. (1962) "A thoerem on duality mappings on Banach spaces", Arkiv Matematik 4: 405-411.

[2] Cioranescu, I. (1990) Geometry of Banach spaces, duality mappings and nonlinear problems. "Mathematics and its Applications" 62, Kluwer Academic Publishers, London.

[3] Chidume, C.E. (1998) Geometric properties of Banach spaces and nonlinear iteractions. I.C.T.P., Trieste.

[4] Day, M.M. (1944) "Uniform convexity in factor and conjugate spaces", Annals of Mathematics 45(2): 375-385.

[5] Day, M.M. (1941) "Reflexive Banach spaces not isomorphic to uniform convex spaces", Bull. Amer. Math. Soc. 47: 313-317.

[6] Diestel, J. (1975) Geometry of Banach spaces. Lec. N. in Math. 485, Springer-Verlag, Berlin.

[7] Lindenstrauss, J.; Tzafriri, L. (1979) Classical Banach Spaces, II. Springer-Verlag, Berlin.

[8] Reich, S. (1981) "On the asymptotic behavior of nonlinear semigroups and the range of accretive operators", J. Math. Anl. Appl. 79: 113-126.

[9] Xu, Z.; Roach, G.F. (1991) "Characteristic inequalities of uniformly convex and uniformly smooth Banach spaces", J. Math. Anal. Appl. 157: 189-210.

[10] Ugalde, W.J. (1998) Characterization of Banach spaces by the duality map. Tesis de Diploma, International Center for Theoretical Physics, Trieste. 